\title{
Magnetic field-induced phase transitions in the antiferromagnet $\mathrm{Nd}_{0.6} \mathrm{Dy}_{0.4} \mathrm{Fe}_{3}\left(\mathrm{BO}_{3}\right)_{4}$
}

\author{
G.A. Zvyagina ${ }^{1}$, K.R. Zhekov ${ }^{1}$, I.V. Bilych ${ }^{1}$, A.A. Zvyagin ${ }^{1,2}$, A.N. Bludov ${ }^{1}$, \\ V.A. Pashchenko ${ }^{1}$, and I.A. Gudim ${ }^{3}$ \\ ${ }^{1}$ B. Verkin Institute for Low Temperature Physics and Engineering of the National Academy of Sciences of Ukraine \\ 47 Lenin Ave., Kharkov 61103, Ukraine \\ E-mail: zvyagina@ilt.kharkov.ua \\ ${ }^{2}$ Max-Planck-Institute for Physics of Complex Systems, 38 Noethnitzer Strasse, Dresden D-01187, Germany \\ ${ }^{3}$ L.V. Kirenskiy Institute of Physics, Siberian Branch of the Russian Academy of Sciences \\ Krasnoyarsk 660036, Russia
}

Received July 13, 2013

\begin{abstract}
Low-temperature studies of elastic and magnetic characteristics of the single crystal $\mathrm{Nd}_{0.6} \mathrm{Dy}_{0.4} \mathrm{Fe}_{3}\left(\mathrm{BO}_{3}\right)_{4}$ have been performed. A transition to the antiferromagnetically ordered state of the magnetic subsystem has been manifested in the temperature behavior of the velocity and attenuation of acoustic modes and magnetization. Spin-reorientation phase transitions, which reveal themselves as anomalies in the behavior of elastic and magnetic characteristics of the crystal in the external magnetic field applied along the axis of the trigonal symmetry of the crystal have been discovered. The phase $H-T$ diagram for $\mathbf{H} \| C_{3}$ has been constructed.
\end{abstract}

PACS: 72.55.+s Magnetoelastic effects;

74.25.Ld Mechanical and acoustical properties, elasticity and ultrasonic attenuation.

Keywords: rare earth ferroborates, magnetoelastic coupling, magnetic phase transitions.

\section{Introduction}

The last decade is characterized by the surge of interest to the synthesis and study of materials, which reveal connection between their magnetic and electric properties [1]. Among those materials are ferroborates of rare earth (RE) elements with the common chemical formula $\mathrm{RFe}_{3}\left(\mathrm{BO}_{3}\right)_{4}$ ( $\mathrm{R}=\mathrm{Y}$; La-Nd; Sm-Er), in several representatives of which the onset of the spontaneous and magnetic fieldinduced electric polarization in the magnetically ordered phase was observed [2].

Features of magnetic and electric properties of such noncenter-symmetric single crystals (trigonal space group $R 32$ ) are determined by the presence of magnetic ions of $d$ and $f$-groups. The interaction in magnetic subsystems essentially depends on the properties of RE ions, which induces variety in the character of the magnetic ordering (all compounds of the present family are antiferromagnets (AF) with the Néel temperature of $30 \mathrm{~K}<T_{N}<40 \mathrm{~K}$ ), the rearrangement of the magnetic structure in the external magnetic field and in the behavior of magnetoelectric polarization. For example, ferroborates of Sm, Er, Nd are magnets with the "easy plane" (EP) magnetic anisotropy, while ferroborates of Pr, Tb, Dy are "easy axis" (EA) AFs. Some compounds transfer spontaneously from the EP to the EA state (e.g., Gd, Ho based ferroborates). Electric polarization appears spontaneously in ferroborates, which have the EP magnetic configuration and magnetic fieldinduced one takes place in EP compounds, as well as in EA ones [2].

To investigate the concurrent contributions of various RE ions to the magnetic anisotropy of ferroborates, and to understand mechanisms of the onset of magnetoelectric polarization, single crystals of ferroborates of binary chemical composition $\mathrm{Nd}_{1-x} \mathrm{Dy}_{x} \mathrm{Fe}_{3}\left(\mathrm{BO}_{3}\right)_{4}$ were synthesized in the L.V. Kirenskiy Institute for Physics of the Siberian branch of RAN, in which for small concentration of Dy a spontaneous spin reorientation from the EP state to the EA state can arise. Really in the single crystal $\mathrm{Nd}_{0.75} \mathrm{Dy}_{0.25} \mathrm{Fe}_{3}\left(\mathrm{BO}_{3}\right)_{4}$ anomalies in the temperature behavior of the magnetic susceptibility at $T_{N}=32 \mathrm{~K}$ and $T_{R}=25 \mathrm{~K}$ [3] were observed; they were connected by the authors with the transition of the crystal to the antiferromagnetic EP state and spin-reorientation transition (from 
the EP state to the EA one), respectively. However, studies of magnetic and magnetoelastic characteristics performed in Refs. 4, 5, have shown that in this compound the spontaneous reorientation $\mathrm{EP} \rightarrow \mathrm{EA}\left(T_{\mathrm{cr}}=16 \mathrm{~K}\right)$ takes place in a composite way, via an intermediate phase $(16 \mathrm{~K}<T<25 \mathrm{~K})$. The magnetic structure of that phase has not been determined finally yet. Nevertheless, basing on the study of the behavior of magnetoelastic characteristics of this compound we have constructed low-temperature regions of phase $H-T$ diagrams for various directions of the external magnetic field [5,6], where we defined lines of registered phase transitions (PT) in temperature as well as in the magnetic field. Phase diagrams have complicated enough structures and they are characterized by the presence of several lines of PTs and, accordingly, several magnetic phases. We have supposed that such complicated phase diagrams can be the manifestation of the interaction between several magnetic sublattices of the crystal, i.e., the compound $\mathrm{Nd}_{0.75} \mathrm{Dy}_{0.25} \mathrm{Fe}_{3}\left(\mathrm{BO}_{3}\right)_{4}$ has to be considered as the multisublattice AF.

It is worthwhile to notice that recent neutron diffraction experiments have shown that in the single crystal of $\mathrm{Nd}$ ferroborate at $T_{\mathrm{cr}}=13 \mathrm{~K}$ the spontaneous transformation of the collinear EP magnetic structure to the incommensurate spiral one takes place [7], and in Dy ferroborate below $20 \mathrm{~K}$ the spontaneous tilt of Dy magnetic moments from the EA direction ( $C_{3}$ axis) has been observed [8]. In such a way, the question about the real magnetic configuration of the intermediate ( $16 \mathrm{~K}<T<25 \mathrm{~K})$ and low-temperature magnetic phases $(T<16 \mathrm{~K})$ in $\mathrm{Nd}_{0.75} \mathrm{Dy}_{0.25} \mathrm{Fe}_{3}\left(\mathrm{BO}_{3}\right)_{4}$ also remains open.

By the present time other representatives of this family of crystals with $x=0.1 ; 0.15 ; 0.25$ have been studied [3,9]. It was shown that the decrease of the parameter $x$ tends to the decreasing of the temperature of the spin reorientation $\mathrm{EP} \rightarrow \mathrm{EA}$.

The present work is devoted to the study of acoustic characteristics of the compound $\mathrm{Nd}_{0.6} \mathrm{Dy}_{0.4} \mathrm{Fe}_{3}\left(\mathrm{BO}_{3}\right)_{4}$, belonging to the family of binary ferroborates $\mathrm{Nd}_{1-x} \mathrm{Dy}_{x} \mathrm{Fe}_{3}\left(\mathrm{BO}_{3}\right)_{4}$. The properties of this compound are not studied well yet. The results of experimental and theoretical studies of temperature and magnetic field behavior of magnetization (magnetic susceptibility) have been presented at the conferences ICYS LTP (Kharkov) and MISM (Moscow) in 2011 $[10,11]$. As a result of the analysis of the experimental data and performed calculations, the authors came to the conclusions that in $\mathrm{Nd}_{0.6} \mathrm{Dy}_{0.4} \mathrm{Fe}_{3}\left(\mathrm{BO}_{3}\right)_{4}$ at low temperatures and $H=0$ the magnetic moments of Nd, Dy and Fe subsystems are oriented along the trigonal axis $C_{3}$ (collinear phase). It means that at $40 \%$ concentration of Dy, the contribution of that subsystem to the magnetic anisotropy becomes crucial and it stabilizes the EA magnetic configuration. The temperature of the magnetic ordering $\left(T_{N}\right)$ was not indicated. The observed jump of the magnetization in the magnetic field applied along the axis $C_{3}\left(H_{s f} \approx 1.9 \mathrm{~T}\right)$ at the tempera- ture $2 \mathrm{~K}$, was interpreted as the manifestation of the PT of the spin-flop type in the Fe subsystem.

Our previous studies have shown that in RE ferroborates the connection between the magnetic and elastic subsystems is strong enough [12,13]. Anomalies in the behavior of elastic characteristics in the vicinities of magnetic phase transformations, as a rule, are very bright. It gives the opportunity to discover such transitions, and also to determine their kind often more reliably than with the help of other, even only magnetic methods [14]. This is why, the ultrasound studies of the compound $\mathrm{Nd}_{0.6} \mathrm{Dy}_{0.4} \mathrm{Fe}_{3}\left(\mathrm{BO}_{3}\right)_{4}$ are in-time and actual.

\section{Technique of the experiment and samples}

Isometric single crystals of $\mathrm{Nd}_{0.6} \mathrm{Dy}_{0.25} \mathrm{Fe}_{3}\left(\mathrm{BO}_{3}\right)_{4}$ have been grown from the dilutions-melts based on bismuth thrimolibdates according the technology described in Ref. 4, and have sizes up to 10-12 mm. We worked with the green transparent crystal representing the hexahedral prism with the height of order of $5 \mathrm{~mm}$ in the direction close to the symmetry axis $C_{3}$. Two experimental samples were cut from different parts of the single crystal, which differed by the shape and size. The sample \#1 was massive enough and had the size $2 \mathrm{~mm}\left(\| C_{3}\right) \times 3 \mathrm{~mm}\left(\| C_{2}\right) \times 2.5 \mathrm{~mm}\left(\perp C_{2}\right)$. The sample \#2 (thick) had the size $2.5 \times 0.5 \times 1.5 \mathrm{~mm}$, respectively. The orientation of the samples was performed by inverse x-ray imaging (Laue method).

Working faces of the samples were polished with the corundum powder. Plane-parallelism of the faces was controlled by the optimeter and was not lower than $0.003^{\circ}$. Running ahead, we remark that the values of critical temperatures and fields observed in the behavior of acoustic characteristics were not different for both experimental samples.

Measurements of the relative changes of the velocity and attenuation of the sound were performed on the automated set-up described in Ref. 15. The precision of the measurements with the thickness of samples $\sim 0.5 \mathrm{~mm}$ was about $\sim 10^{-4}$ for the velocity and $\sim 0.05 \mathrm{~dB}$ for the attenuation. We have studied the behavior of the velocity and attenuation of transverse acoustic modes depending on temperature and magnetic field. The range of the temperature was $1.7-120 \mathrm{~K}$, and of the external magnetic field was up to $5.5 \mathrm{~T}$.

As a result of acoustic investigations we have observed anomalies in the behavior of magnetoelastic characteristics of the crystal $\mathrm{Nd}_{0.6} \mathrm{Dy}_{0.4} \mathrm{Fe}_{3}\left(\mathrm{BO}_{3}\right)_{4}$, which, in our opinion, are related to magnetic phase transformations in the considered compound. To check this assumption we have performed measurements of the magnetization of the crystal as a function of temperature in the range $5-300 \mathrm{~K}$ and the external magnetic field up to $5 \mathrm{~T}$ oriented along the $C_{3}$ axis with the help of the SQUID magnetometer MPMS-XL. The same sample \#2 was used for those measurements as in the acoustic experiments. 


\section{Results and discussion}

The temperature behavior of the transverse ${ }^{\star}$ acoustic modes has manifested several anomalies in the interval 1.7-120 K, Fig. 1. Below they are listed for the temperatures decreasing.

1. The softening of the velocity of some modes $\left(C_{44}\right.$ mode $^{\star \star}$ and the mode $(\mathbf{q}\|\mathbf{y}, \mathbf{u}\| \mathbf{z})$ ), starting from the temperature $T^{*} \approx 100 \mathrm{~K}$, and the kink at $T^{* *} \approx 60 \mathrm{~K}$. The velocity of the $C_{66}$ mode is softed starting from $T^{* *}$.

2 . The jump of the velocity (the maximum in the attenuation) at $T=31.4 \mathrm{~K}$.

First let us discuss the anomaly at $T=31.4 \mathrm{~K}$. We observed analogous behavior of acoustic characteristics in $\mathrm{NdFe}_{3}\left(\mathrm{BO}_{3}\right)_{4}$ [14] and in the binary compound $\mathrm{Nd}_{0.75} \mathrm{Dy}_{0.25} \mathrm{Fe}_{3}\left(\mathrm{BO}_{3}\right)_{4}$ in the vicinities of the Néel temperature $[5,6]$. Thus, we can suppose according to the characteristic shape of that anomaly that in $\mathrm{Nd}_{0.6} \mathrm{Dy}_{0.4} \mathrm{Fe}_{3}\left(\mathrm{BO}_{3}\right)_{4}$ the temperature $31.4 \mathrm{~K}$ is the magnetic ordering point.

To understand which of observed features in the elastic properties have magnetic nature, we have studied the temperature behavior of the magnetization of this crystal. The dependence of the magnetization was measured in the weak field $H=0.02 \mathrm{~T}$, oriented in the direction along to the $C_{3}$ axis; it is shown in Fig. 1. As we can see, it has two maxima at the temperature values 31.4 and $19 \mathrm{~K}$, respectively.

It is known that parent compounds $\mathrm{NdFe}_{3}\left(\mathrm{BO}_{3}\right)_{4}[16]$ and $\mathrm{DyFe}_{3}\left(\mathrm{BO}_{3}\right)_{4}$ [17] demonstrate the maximum at $T_{N}$ in the temperature behavior of the magnetization (magnetic susceptibility). At the same time all studied until now crystals from the family $\mathrm{Nd}_{1-x} \mathrm{Dy}_{x} \mathrm{Fe}_{3}\left(\mathrm{BO}_{3}\right)_{4}$ do not reveal such an anomaly at $T_{N}$, but have maxima, related to spontaneous spin-reorientation transitions $[3,4,9]$.

We can determine the temperature $T_{N}=31.4 \mathrm{~K}$ as the magnetic ordering point in $\mathrm{Nd}_{0.6} \mathrm{Dy}_{0.4} \mathrm{Fe}_{3}\left(\mathrm{BO}_{3}\right)_{4}$ on the basis of two facts:

1. The higher-temperature maximum in the temperature behavior of the magnetization and the characteristic jump of the sound velocities are observed at the same temperature;

2. There are no any additional anomalies in the elastic characteristics below $31.4 \mathrm{~K}$, which indicate spontaneous spin reorientation, as for example in the $\mathrm{Nd}_{0.75} \mathrm{Dy}_{0.25} \mathrm{Fe}_{3}\left(\mathrm{BO}_{3}\right)_{4}$.

In addition to the anomaly at $31.4 \mathrm{~K}$, the temperature dependence of the magnetization of the studied crystal manifests the broad maximum at lower temperature. Similar maxima were observed in the temperature behavior of magnetization of dysprosium-based [17] and neodimium-

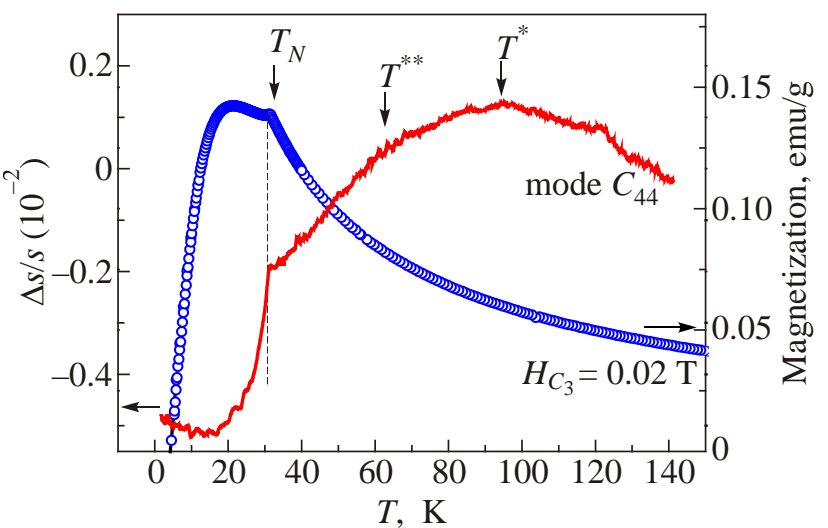

Fig. 1. (Color online) The temperature dependence of the behavior of the sound velocity of the transverse mode $C_{44}\left(\mathbf{q}\|\mathbf{z}\| C_{3}\right.$, $\mathbf{u} \| \mathbf{x} \perp C_{2}$ ) of the $\mathrm{Nd}_{0.6} \mathrm{Dy}_{0.4} \mathrm{Fe}_{3}\left(\mathrm{BO}_{3}\right)_{4}$ single crystal. The temperature dependence of the magnetization measured in $H=0.02 \mathrm{~T}$ applied along $C_{3}$ is also plotted.

based ferroborates below $T_{N}$ [16]. The authors connected those maxima with the Schottky-type anomalies. If it is correct, than large enough amount of magnetic ions do not take part in the establishing of the magnetic order.

Notice that the temperature behavior of the magnetization does not show any features at temperatures $T^{*} \approx 100 \mathrm{~K}$ and $T^{* *} \approx 60 \mathrm{~K}$. Features in the behavior of acoustic characteristics at those temperatures can be connected, for example, with structural deformations, caused by relatively large content of dysprosium. To remind, at the temperature $280 \mathrm{~K}$ the structure PT of the first order $\left(R 32 \rightarrow P 3_{1} 21\right)$ is realized in dysprosium ferroborate [17].

Magnetic field dependences of the velocities and attenuations of transverse modes are studied at fixed temperature values in the range $1.7-35 \mathrm{~K}$ for three directions of the external magnetic field: $\mathbf{H} \| C_{3}$, and $\mathbf{H} \| C_{2}, \mathbf{H} \perp C_{2}$ (field in the basal plane).

\subsection{Field along the trigonal axis $C_{3}(\mathbf{H} \| \mathbf{z})$}

In the magnetic field dependences of the velocities of all studied transverse modes in the fields, respectively, $H_{\mathrm{cr} 1}$ and $H_{\mathrm{cr} 2}$ two closely situated features (jumps) were discovered, which followed by the small hysteresis $(0.08 \mathrm{~T})$. Anomalies in the attenuation are related to the jumps in the velocity. The critical fields $H_{\mathrm{cr} 1}$ and $H_{\mathrm{cr} 2}$ are determined as average values between the positions of the anomalies in the velocity (attenuation) for growing and decrease of the value of the magnetic field. The example of the magnetic field dependence of the velocity and attenuation of the mode $\mathbf{q}\|\mathbf{y}, \mathbf{u}\| \mathbf{z}$ for the lowest temperature of the experi-

* It was determined earlier [12-14], that transverse modes demonstrate the brightest features in the behavior of the elastic characteristics of ferroborates. The effects in longitudinal modes are one order of magnitude weaker, and, hence, will not be analyzed here.

** In figures we use the following notations: $\Delta s / s$ and $\Delta \alpha$ are relative changes of the velocity and attenuation of sound waves ( $\mathbf{q}$ is the wave vector, $\mathbf{u}$ is the polarization), spreading along $x, y, z$ axes of the standard for the trigonal crystal set of Cartesian coordinates $\left(y\left\|C_{2}, z\right\| C_{3}\right)$. 

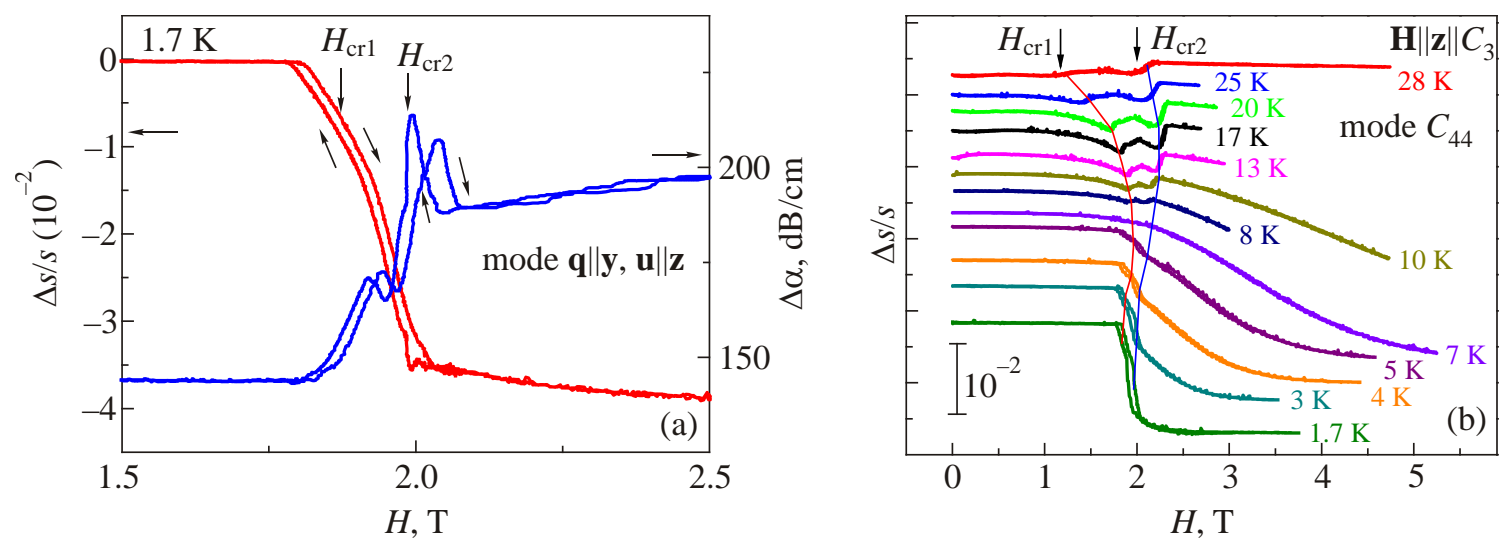

Fig. 2. (Color online) Magnetic field dependence of the behavior of the acoustic characteristics of the $\mathrm{Nd}_{0.6} \mathrm{Dy}_{0.4} \mathrm{Fe}_{3}\left(\mathrm{BO}_{3}\right)_{4}$ in the magnetic field $\mathbf{H} \| C_{3}$ : (a) velocity and attenuation of the acoustic mode ( $\mathbf{q}\left\|C_{2}\right\| \mathbf{y}, \mathbf{u}\left\|C_{3}\right\| \mathbf{z}$ ) at the temperature $1.7 \mathrm{~K}$; (b) velocity of the acoustic mode $C_{44}$ at various temperatures in the range $1.7 \mathrm{~K} \leq T \leq T_{N}$.

ment $1.7 \mathrm{~K}$ is presented in Fig. 2(a). The increase of the temperature somewhat changes the scale and shape of anomalies, and the value of the field $H_{\mathrm{cr} 2}$ is almost the same, but the value $H_{\mathrm{cr} 1}$ is shifted to the lower values of the field, Fig. 2(b). We register the features in the velocity and attenuation of the sound at $H_{\mathrm{cr} 1}$ and $H_{\mathrm{cr} 2}$ in the temperature range from the lowest temperature of the experiment $1.7 \mathrm{~K}$ till $30 \mathrm{~K}$, i.e., practically till $T_{N}$.

We have supposed that observed anomalies in the behavior of magnetoelastic characteristics of the crystal $\mathrm{Nd}_{0.6} \mathrm{Dy}_{0.4} \mathrm{Fe}_{3}\left(\mathrm{BO}_{3}\right)_{4}$ can be caused by phase transformations in its magnetic subsystem. To check that assumption the behavior of the magnetization of the crystal as a function of the external magnetic field up to $5 \mathrm{~T}$, for several fixed temperatures from the range $T \leq T_{N}$ were performed. Step-like anomalies in the magnetization curves of the magnetic field dependence at temperatures 5, 7, 10 and $20 \mathrm{~K}$ were observed Fig. 3(a). Values of the critical fields for anomalies in magnetoacoustic and magnetic dependences well correlate, Fig. 3(b). Note that previous measurements of the magnetization behavior in the field $\mathbf{H} \| C_{3}$, presented in Refs. 10, 11, have observed only one jump, which was interpreted as the spin-flop transition of the magnetic sublattices of iron.

Hence, it is possible to conclude that observed anomalies in the magnetic field dependences of the sound characteristics and magnetization are caused by several magnetic structure transitions, that testifies large number of interacting magnetic sublattices.

Discovered magnetic PTs reveal themselves as anomalies at the temperatures $T_{c 1.2}$ and in temperature dependences of the sound velocity and attenuation at the fixed value of the external magnetic field $\mathbf{H} \| C_{3}$ (Fig. 4).

\subsection{Field in the basal plane $\left(\mathbf{H} \| C_{2}, \mathbf{H} \perp C_{2}\right)$}

Magnetic field up to $5 \mathrm{~T}$ applied in the basal plane of the crystal does not change the form of the temperature dependence of the sound velocity and attenuation. As for the case $H=0$, one can see only one anomaly in curves, which is connected with the magnetic ordering point (Fig. 4).

Magnetic field behavior of acoustic characteristics at the fixed temperature $T \leq T_{N}$ was investigated for the cases $\mathbf{H} \| C_{2}$ and $\mathbf{H} \perp C_{2}$. The example of the dependences of
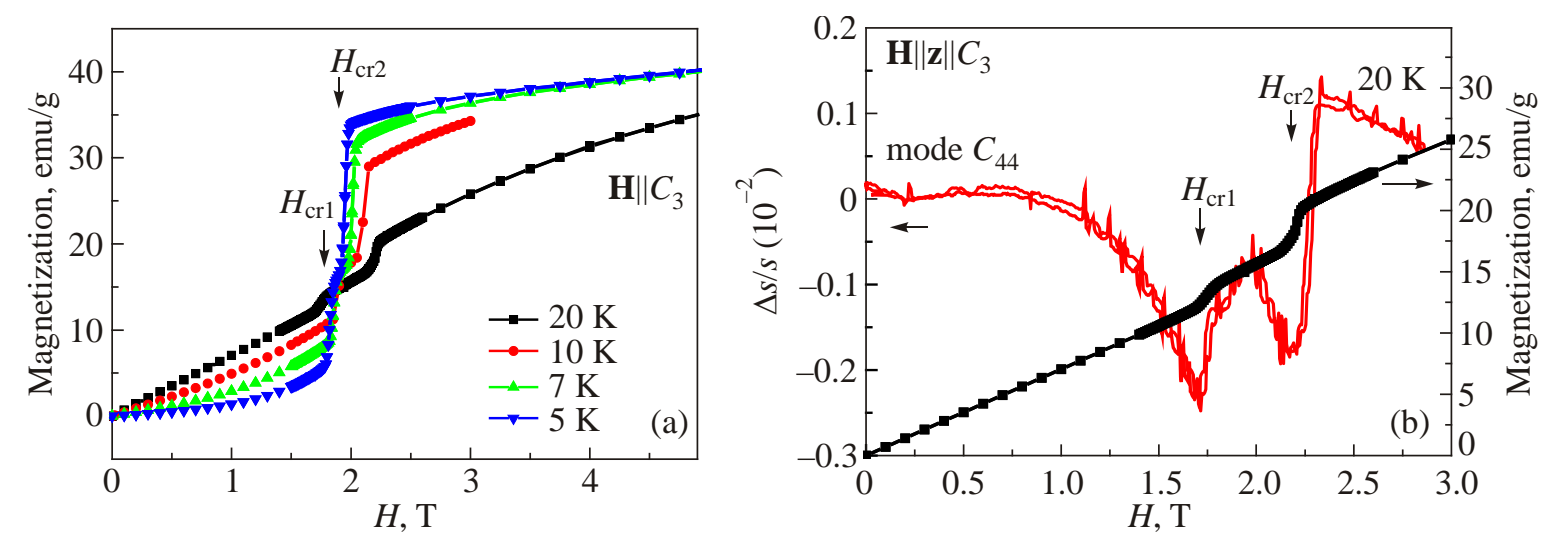

Fig. 3. (Color online) (a) The magnetic field dependences of the magnetization of the $\mathrm{Nd}_{0.6} \mathrm{Dy}_{0.4} \mathrm{Fe}_{3}\left(\mathrm{BO}_{3}\right)_{4}$ measured along $C_{3}$ at the various temperatures: 5; 7; 10 and $20 \mathrm{~K}$. The positions of the $H_{\mathrm{cr} 1.2}$ are indicated for $5 \mathrm{~K}$. (b) The magnetic field dependences of the velocity of the $C_{44}$ mode and the magnetization at $20 \mathrm{~K}$ in the magnetic field $\mathbf{H} \| C_{3}$. 


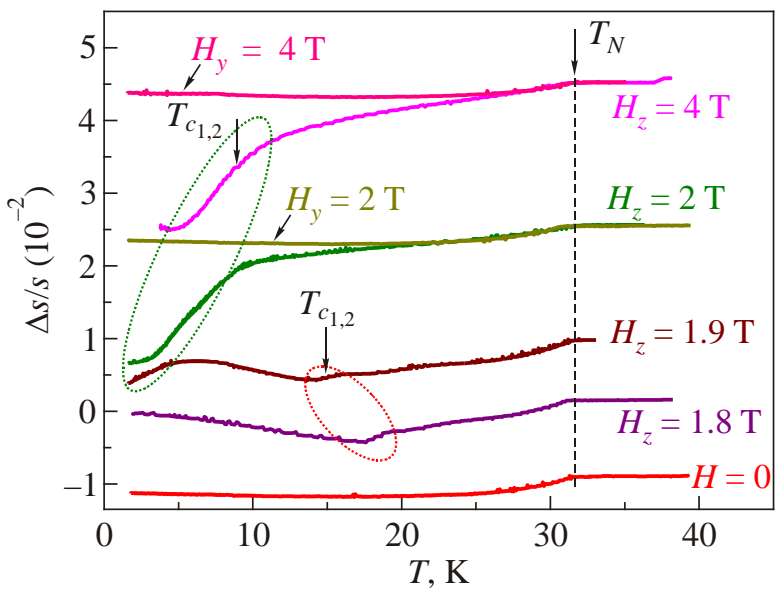

Fig. 4. (Color online) Temperature behavior of the velocity of the acoustic $C_{44}$ mode in $H=0$ and in various external magnetic fields $\mathbf{H} \| C_{3}(H=1.8,1.9,2$, and $4 \mathrm{~T})$ and $\mathbf{H} \| C_{2}(H=2$ and $4 \mathrm{~T})$.

the velocity of the acoustic mode $C_{66}$ at $T=5 \mathrm{~K}$ for $\mathbf{H} \| C_{2}$ and $\mathbf{H} \perp C_{2}$ is presented in Fig. 5. For comparison in the same figure the dependence of the velocity of that mode for $\mathbf{H} \| C_{3}$, demonstrating jumps at $H_{\mathrm{cr} 1}$ and $H_{\mathrm{cr} 2}$ is presented. As we can see from the figure, a small anisotropy is present for the velocity for two mutually perpendicular directions of the field in the basal plane. In the case $\mathbf{H} \| C_{2}$ the velocity increases steadily with the growth of the field, and for $\mathbf{H} \perp C_{2}$ its value practically does not depend on the field. In both cases we did not register any anomalies, which could be evidence of phase transformations in the magnetic subsystem of the crystal.

We did not perform measurements of the field dependences of the magnetization of the compound in the magnetic field applied in the basic plane. Such measurements for the case $\mathbf{H} \| C_{2}$ (field up to $9 \mathrm{~T}$ ) in the temperature range $2-40 \mathrm{~K}$ were performed in the L.V. Kirenskiy Institute for Physics and were discussed in Refs. 10, 11. Those dependences did not reveal seen anomalies. For each value of the temperature only monotonous growth of the magnet-

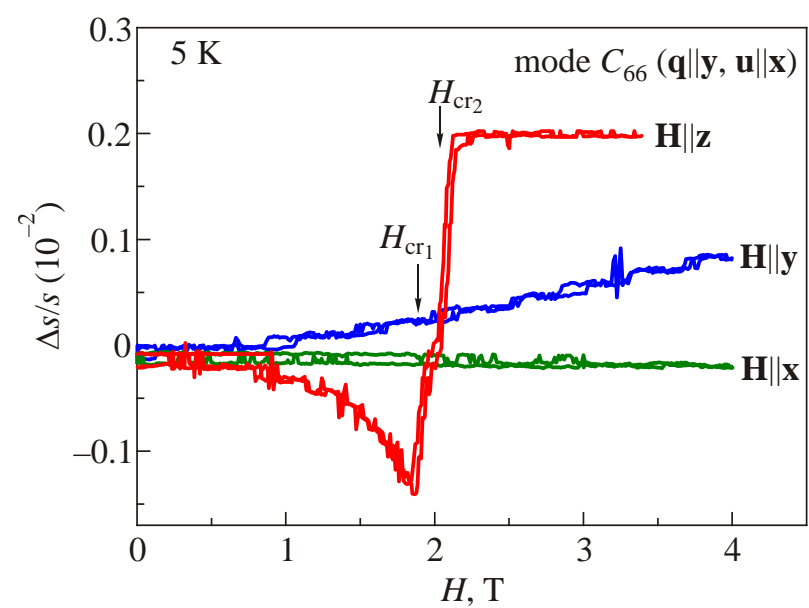

Fig. 5. (Color online) Magnetic field dependences of the velocity of the $C_{66}$ acoustic mode $(\mathbf{q}\|\mathbf{y}, \mathbf{u}\| \mathbf{x})$ at $5 \mathrm{~K}$ in external magnetic fields applied along $\mathbf{H}\left\|C_{3}, \mathbf{H}\right\| C_{2}$, and $\mathbf{H} \perp C_{2}$. ization in the total studied range of field values was registered, testifying absence of field $\mathbf{H} \perp C_{3}$ induced reorientation PTs in the crystal.

One can see correlation in the behavior of acoustic and magnetic characteristics of the crystal in the external magnetic field in the case $\mathbf{H} \| C_{3}$, as well as for the field applied in the basal plane (at least for the case $\mathbf{H} \| C_{2}$ ).

\section{Summary}

Hereby, as the result of the study of the behavior of acoustic and magnetic characteristics of the ferroborate $\mathrm{Nd}_{0.6} \mathrm{Dy}_{0.4} \mathrm{Fe}_{3}\left(\mathrm{BO}_{3}\right)_{4}$ new phase transitions were discovered, which were induced by the external magnetic field $\mathbf{H} \| C_{3}$. We have constructed the low-temperature region of the phase $H-T$ diagram of that compound for the case $\mathbf{H} \| C_{3}$ (Fig. 6). The diagram, as the one for the case of $\mathrm{Nd}_{0.75} \mathrm{Dy}_{0.25} \mathrm{Fe}_{3}\left(\mathrm{BO}_{3}\right)_{4}$, is characterized by several lines of phase transitions, and, accordingly, of several magnetic phases. In principle, the diagrams of these related compounds are similar. The analogous low-temperature behavior of those systems is caused, naturally, by the close chemical contents. That similarity reveals itself, as we have shown, in the features of the behavior of their magnetic and acoustic characteristics in nonzero magnetic field. At the same time, in zero magnetic field for $\mathrm{Nd}_{0.6} \mathrm{Dy}_{0.4} \mathrm{Fe}_{3}\left(\mathrm{BO}_{3}\right)_{4}$ spontaneous spin-reorientation transitions are absent; at $H=0$ below the temperature of magnetic ordering the system probably is in practically "easy-axis" magnetic state. Apparently the large concentration of Dy ions suppresses the EP anisotropy, caused by neodymium, and does not permit to realize either “easy-plane” or intermediate phases. The external magnetic field, applied along the trigonal symmetry axis $\mathrm{C}_{3}$ perhaps promotes the onset of mentioned phases.

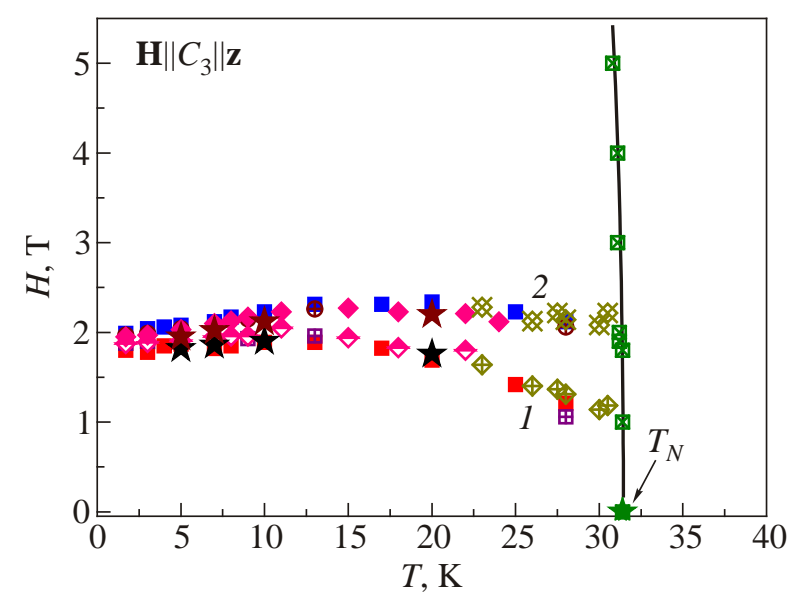

Fig. 6. (Color online) Fragment of $H-T$ diagram of the compound $\mathrm{Nd}_{0.6} \mathrm{Dy}_{0.4} \mathrm{Fe}_{3}\left(\mathrm{BO}_{3}\right)_{4}$ in magnetic fields applied along $C_{3}$ axis of the crystal. Tetragons indicate the positions of features in temperature and magnetic field dependencies of the velocity and absorption for various acoustic modes. Stars indicate the positions of features in the temperature and magnetic field dependences of the magnetization. 
1. W. Erenstein, N.D. Mathur, and J.F. Scott, Nature Mater. 442, 759 (2006).

2. A.M. Kadomtseva, Yu.F. Popov, G.P. Vorob’ev, A.P. Pyatakov, S.S. Krotov, P.I. Kamilov, V.Yu. Ivanov, A.A. Mukhin, A.K. Zvezdin, L.N. Bezmaternykh, I.A. Gudim, and V.L. Temerov, Fiz. Nizk. Temp. 36, 640 (2010) [Low Temp. Phys. 36, 511 (2010)].

3. Yu.F. Popov, A.M. Kadomtseva, G.P. Vorob’ev, A.A. Mukhin, V.Yu. Ivanov, A.M. Kuz'menko, A.S. Prokhorov, L.N. Bezmaternykh, and V.L. Temerov, Pis'ma Zh. Eksp. Teor. Fiz. 89, 405 (2009).

4. I.A. Gudim, E.V. Eremin, and V.L. Temerov, J. Cryst. Growth 312, 2427 (2010).

5. G.A. Zvyagina, K.R. Zhekov, A.A. Zvyagin, I.V. Bilych, L.N. Bezmaternykh, and I.A. Gudim, Fiz. Nizk. Temp. 36, 352 (2010) [Low Temp. Phys. 36, 279 (2010)].

6. G.A. Zvyagina, K.R. Zhekov, A.A. Zvyagin, I.A. Gudim, and I.V. Bilych, Fiz. Nizk. Temp. 38, 571 (2012) [Low Temp. Phys. 38, 446 (2012)].

7. M. Janoschek, P. Fischer, J. Schefer, B. Roessli, V.M. Meven, V. Petricek, G. Petrakovskii, and L. Bezmaternikh, Phys. Rev. B 81, 094429 (2010).

8. C. Ritter, A. Pankrats, I. Gudim, and A. Vorotynov, J. Phys.: Conf. Series 340, 012065 (2012).
9. A.A. Demidov, I.A. Gudim, and E.V. Eremin, Zh. Eksp. Teor. Fiz. 141, 294 (2012).

10. A.A. Demidov, I.A. Gudim, and E.V. Eremin, Abstracts of ICYS LTP-2011, Kharkiv, Ukraine, p. 83.

11. A.A. Demidov, I.A. Gudim, and E.V. Eremin, Abstracts of MISM-2011, Moscow, Russia, p. 477.

12. G.A. Zvyagina, K.R. Zhekov, L.N. Bezmaternykh, I.A. Gudim, I.V. Bilych, and A.A. Zvyagin, Fiz. Nizk. Temp. 34, 1142 (2008) [Low Temp. Phys. 34, 901 (2008)].

13. G.A. Zvyagina, K.R. Zhekov, A.A. Zvyagin, I.V. Bilych, L.N. Bezmaternykh, and I.A. Gudim, Fiz. Nizk. Temp. 36, 376 (2010) [Low Temp. Phys. 36, 296 (2010)].

14. G.A. Zvyagina, K.R. Zhekov, I.V. Bilych, A.A. Zvyagin, I.A. Gudim, and V.L. Temerov, Fiz. Nizk. Temp. 37, 1269 (2011) [Low Temp. Phys. 37, 1010 (2011)].

15. E.A. Masalitin, V.D. Fil', K.R. Zhekov, A.N. Zholobenko, and T.V. Ignatova, Fiz. Nizk. Temp. 29, 93 (2003) [Low Temp. Phys. 29, 72 (2003)].

16. N. Tristan, R. Klingeler, C. Hess, B. Buchner, E. Popova, I.A. Gudim, and L.N. Bezmaternykh, J. Magn. Magn. Mater. 316, e621 (2007).

17. E.A. Popova, N. Tristan, A.N. Vasiliev, V.L. Temerov, L.N. Bezmaternykh, N. Leps, B. Buchner, and R. Klingeler, Eur. Phys. J. B 62, 123 (2008). 